\section{SARS-CoV-2 Sequence Analysis during COVID-19 Case Surge, Liberia, 2021}

\author{
Bode Shobayo, ${ }^{1}$ Mitali Mishra, ${ }^{1}$ Stephen Sameroff, \\ Alexandra Petrosov, James Ng, Alper Gokden, \\ Jane MaCauley, Komal Jain, Courtney Renken, \\ James Tanu Duworko, Moses Badio, \\ Wilhemina Jallah, Lisa Hensley, Thomas Briese, \\ W. Ian Lipkin, Nischay Mishra
}

\begin{abstract}
Author affiliations: National Public Health Institute of Liberia, Monrovia, Liberia (B. Shobayo, J. MaCauley); Partnership for Research on Infectious Diseases in Liberia, Monrovia (B. Shobayo, C. Renken, J.T. Duworko, M. Badio, L. Hensley); Columbia University, New York, New York, USA (M. Mishra, S. Sameroff, A. Petrosov, J. Ng, A. Gokden, K. Jain, T. Briese, W.I. Lipkin, N. Mishra); National Institutes of Health, Bethesda, Maryland, USA (C. Renken, L. Hensley); Ministry of Health, Monrovia (W. Jallah)
\end{abstract}

\section{DOI: https://doi.org/10.3201/eid2712.211818}

In June 2021, severe acute respiratory syndrome coronavirus 2 (SARS-CoV-2) cases surged in Liberia. SARSCoV-2 sequences from patients hospitalized during March-July 2021 revealed the Delta variant was in Liberia in early March and was dominant in June, irrespective of geography. Mutations and deletions suggest multiple SARS-CoV-2 Delta variant introductions.

$\mathrm{B}$ efore May 2021, Liberia reported $<10$ coronavirus disease (COVID-19) cases per day among its population of $\approx 5$ million (1). Thereafter, case numbers, hospitalizations, and deaths rapidly increased and peaked to $>200$ cases and 10-15 deaths per day in mid-July 2021 (Appendix Figure 1, https:/ / wwwnc. cdc.gov/EID/article/27/12/21-1818-App1.pdf). To determine whether the rapid case surge was associated with the introduction of severe acute respiratory syndrome coronavirus 2 (SARS-CoV-2) variants of concern or newly emerging variants, we collected nasopharyngeal swab samples from 267 hospitalized patients countrywide during March-July 2021 for high-throughput sequencing.

We collected samples in viral transport media from Bomi, Bong, Grand Cape Mount, Lofa, Margibi, Maryland, Montserrado, and Nimba Counties (Appendix Figure 2). We noted sample collection date and site and sex and median age of patients from whom samples were obtained (Table; Appendix

${ }^{1}$ These first authors contributed equally to this article.
Table). We used Buffer AVL (QIAGEN, https:// www.qiagen.com) lysis buffer to extract total nucleic acid and performed PCR by using the Triplex-CIISARS-Cov-2 rRT PCR assay (2). We conducted further high-throughput sequencing on 89/267 (33.3\%) samples that had cycle threshold values <33 (Appendix Table).

To prepare libraries, we used the Kapa Hyperplus Kit (Roche, https://www.roche.com) on first strand cDNA synthesized from 89 RNA samples (3), then we enriched for SARS-CoV-2 by using myBaits Custom RNA-Seq Kit (Daicel Arbor Biosciences, https://arborbiosci.com). We sequenced captured libraries on Nextseq 2000 or Nextseq 550 (Illumina, https:/ / www.illumina.com), which yielded 5-8 million 220-bp reads per sample. We mapped reads to a SARS-CoV-2 reference sequence (GenBank accession no. NC_045512) to determine variants (Table; Appendix Table).

Of the 89 RNA samples, 77 (86.5\%) yielded complete coding sequences with a minimum depth of $\approx 15 \times$ (GISAID accession nos. EPI_ISL_3547663-705, EPI_ISL_3560291, and EPI_ISL_4232122-52). Using high-throughput sequencing data, we generated consensus fasta sequences of 77 SARS-CoV-2 genomic sequences and further analyzed sequences by using Geneious R10 (https://www.geneious.com), NextStrain (4), and GISAID (5).

Among 77 genomes recovered, 4 (5.2\%) were Alpha variant (B.1.1.7); 6 (7.8\%) were Beta variant (B.1.351); 1 (1.3\%) was Iota variant (B.1.526); 6 (7.8\%) were Eta variant (B.1.525); and 56 (72.7\%) were Delta variant (B.1.617.2) viruses (Table). We identified Delta variant viruses in samples collected in early March and in April and May 2021, from Bong County. Delta variant viruses were co-circulating with Alpha, Beta, Eta, Iota, and other $20 \mathrm{~B}$ variant viruses in Liberia. All 44 sequences recovered during June-July 2021 were from Delta variant viruses (Table). We used complete polyprotein coding sequences from Liberia, other representative SARS-CoV-2 sequences, and variant reference sequences to create a maximum-likelihood, nucleotide-based phylogenetic tree in MEGA X (6) (Figure).

Using reference sequence NC_045512 as a baseline, we found 3 Alpha variant-specific amino acid deletions (H69del, V70del, Y144del) in the surface glycoprotein of all Alpha variant genomes and 3 Beta variant-specific amino acid deletions (L241del, L242del, A243del) in the surface glycoprotein of all Beta variant genomes. All 56 Delta variant genomes had the 2 variant-specific amino acid deletions, F157del and R158del, and 8 of 9 other Delta variant- 
Table. Characteristics of 77 clinical samples collected before and during COVID-19 case surge that yielded complete SARS-CoV-2 coding genomic sequences, Liberia, 2021

\begin{tabular}{|c|c|c|c|c|c|c|c|c|c|c|c|}
\hline \multirow[b]{2}{*}{$\begin{array}{l}\text { Month } \\
\text { collected }\end{array}$} & \multirow[b]{2}{*}{$\begin{array}{l}\text { Total no. } \\
\text { samples }\end{array}$} & \multirow[b]{2}{*}{$\begin{array}{l}\text { Patient } \\
\text { sex, no. }\end{array}$} & \multirow[b]{2}{*}{$\begin{array}{c}\text { Average age, } \\
\text { y (SD) }\end{array}$} & \multirow[b]{2}{*}{ County } & \multirow{2}{*}{$\begin{array}{l}\text { No. } \\
\text { samples/ } \\
\text { county }\end{array}$} & \multicolumn{6}{|c|}{ SARS-CoV-2 variant, no. of samples/county/mo } \\
\hline & & & & & & $\begin{array}{c}\text { Delta } \\
\text { B.1.617.2 }\end{array}$ & $\begin{array}{c}\text { Alpha } \\
\text { B.1.1.7 }\end{array}$ & $\begin{array}{c}\text { Beta } \\
\text { B.1.351 }\end{array}$ & $\begin{array}{c}\text { Eta } \\
\text { B.1.525 }\end{array}$ & $\begin{array}{c}\text { lota } \\
\text { B.1.526 }\end{array}$ & $\begin{array}{l}20 \mathrm{~B} \\
\text { other }\end{array}$ \\
\hline Mar & 4 & $2 \mathrm{M}, 2 \mathrm{~F}$ & $39.25(6.05)$ & $\begin{array}{l}\text { Montserrado } \\
\text { Bong }\end{array}$ & $\begin{array}{l}3 \\
1\end{array}$ & 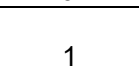 & & 1 & 1 & 1 & \\
\hline Apr & 11 & 10M, 1F & $42.54(11.52)$ & $\begin{array}{c}\text { Montserrado } \\
\text { Grand Cape } \\
\text { Mount }\end{array}$ & $\begin{array}{c}10 \\
1\end{array}$ & 4 & 1 & $\begin{array}{l}3 \\
1\end{array}$ & 2 & & \\
\hline May & 18 & 9M, 9F & $40.11(16.82)$ & $\begin{array}{c}\text { Bong } \\
\text { Margibi } \\
\text { Montserrado } \\
\text { Nimba }\end{array}$ & $\begin{array}{c}1 \\
1 \\
14 \\
2 \\
\end{array}$ & 1 & 3 & 2 & 2 & & $\begin{array}{l}1 \\
2\end{array}$ \\
\hline Jun & 36 & $13 \mathrm{M}, 23 \mathrm{~F}$ & $39.22(18.36)$ & $\begin{array}{c}\text { Lofa } \\
\text { Margibi } \\
\text { Maryland } \\
\text { Montserrado }\end{array}$ & $\begin{array}{c}5 \\
1 \\
1 \\
29\end{array}$ & $\begin{array}{c}5 \\
1 \\
1 \\
29\end{array}$ & & & & & \\
\hline Jul & 8 & $4 \mathrm{M}, 4 \mathrm{~F}$ & $51.25(9.71)$ & $\begin{array}{c}\text { Margibi } \\
\text { Montserrado } \\
\text { Nimba }\end{array}$ & $\begin{array}{l}1 \\
5 \\
2\end{array}$ & $\begin{array}{l}1 \\
5 \\
2\end{array}$ & & & & & \\
\hline
\end{tabular}

*Liberia experienced a surge in COVID-19 cases during June 2021. Blank cells indicate no variants detected. COVID-19, coronavirus disease; SARSCoV-2, severe acute respiratory syndrome coronavirus 2.

specific amino acid substitutions in the surface glycoprotein (T19R, G142D, E156G, L452R, T478K, D614G, P681R, and D950N). The A222V surface glycoprotein mutation was absent in only 2/56 Delta variant genomes, LIB-0226 and LIB-0217, collected from Monteserrado County in May 2021 (4). We observed another mutation in the surface glycoprotein, V367L, in 14 sequences: 1 from Bong, 2 from Margibi, 1 from Maryland, 9 from Montserrado, and 1 from Nimba. No sequences recovered from Lofa County had the V367L mutation. We noted the R724K mutation in the open reading frame 1 a region of 2 sequences from Lofa, LIB-0131 and LIB-0133. LIB-0073 and LIB-0093 sequences collected from Montserrado County had 2 amino acid deletions in the open reading frame 8 region (position 120-121).

Recent surges in COVID-19 in many countries have been associated with the emergence of highly transmissible Delta variant viruses $(7,8)$. In March 2021, the National Public Health Institute of Liberia sequenced 10 random samples from hospitalized COVID-19 patients in Monteserrado; all sequences were Alpha variant viruses (B. Shobayo, unpub. data).

A limitation of our study is the small sample sets used for analysis; nonetheless, our findings suggest that Alpha and other circulating variant viruses were replaced by Delta variant viruses countrywide in Liberia in $<3$ months. Mutation and phylogenetic analyses further indicate that several Delta variant strains were circulating after March 2021 and suggest multiple separate introductions.

Before June 2021, only a small percentage of the population was vaccinated in Liberia. The infections we report occurred in unvaccinated persons. The
Ministry of Health, Liberia, initiated a vaccination drive in August 2021. By September, $\approx 130,000$ persons, $>2 \%$ of the population, had received a single dose of the Johnson \& Johnson/Janssen vaccine (https://www.jnj.com). The COVID-19 vaccination campaign is ramping up as $<30$ cases/day are reported in Liberia, but the currently circulating Delta variants are a concern because they contain mutations and deletions in the surface glycoprotein that might influence vaccine efficacy (9). Liberia should continued surveillance for SARS-CoV-2 variants of concern to determine whether additional vaccination or public health measures are needed to curb severe disease and future case surges in the country.

\section{Acknowledgments}

We thank Pryanka Sharma and Gilbert Smith; Melvin Johnson, John Fayiah and Julie Blie and all the members of Partnership for Research on Vaccines and Infectious Diseases in Liberia; Julius Teahton, Francis Jaryan, John Dogba, Fahn Taweh, and Joseph Tahyor; and National Public Health Institute of Liberia research and lab team members for their support in collection and transport of samples.

Financial support was provided by by the Skoll Foundation for the Global Alliance for Pandemic Prevention (GAPP grant no. 20-45017), the Tong Tsung and Wei Fong Chao Foundation (grant no. GT007457), and the Chau Hoi Sheun Foundation (grant no. GT007457).

The National Institute of Allergy and Infectious Diseases, National Institutes of Health, also participated in study design, analysis and interpretation of data, writing of the report, and decision to submit the article for publication. 


\section{About the Author}

Dr. Shobayo is public health and medical research scientist and deputy director at National Public Health Institute of Liberia, Monrovia, Liberia, and member of Partnership for Research on Infectious Diseases in Liberia. His research interests include survillence and investigation of emerging and re-emerging viruses and their impacts on public health.

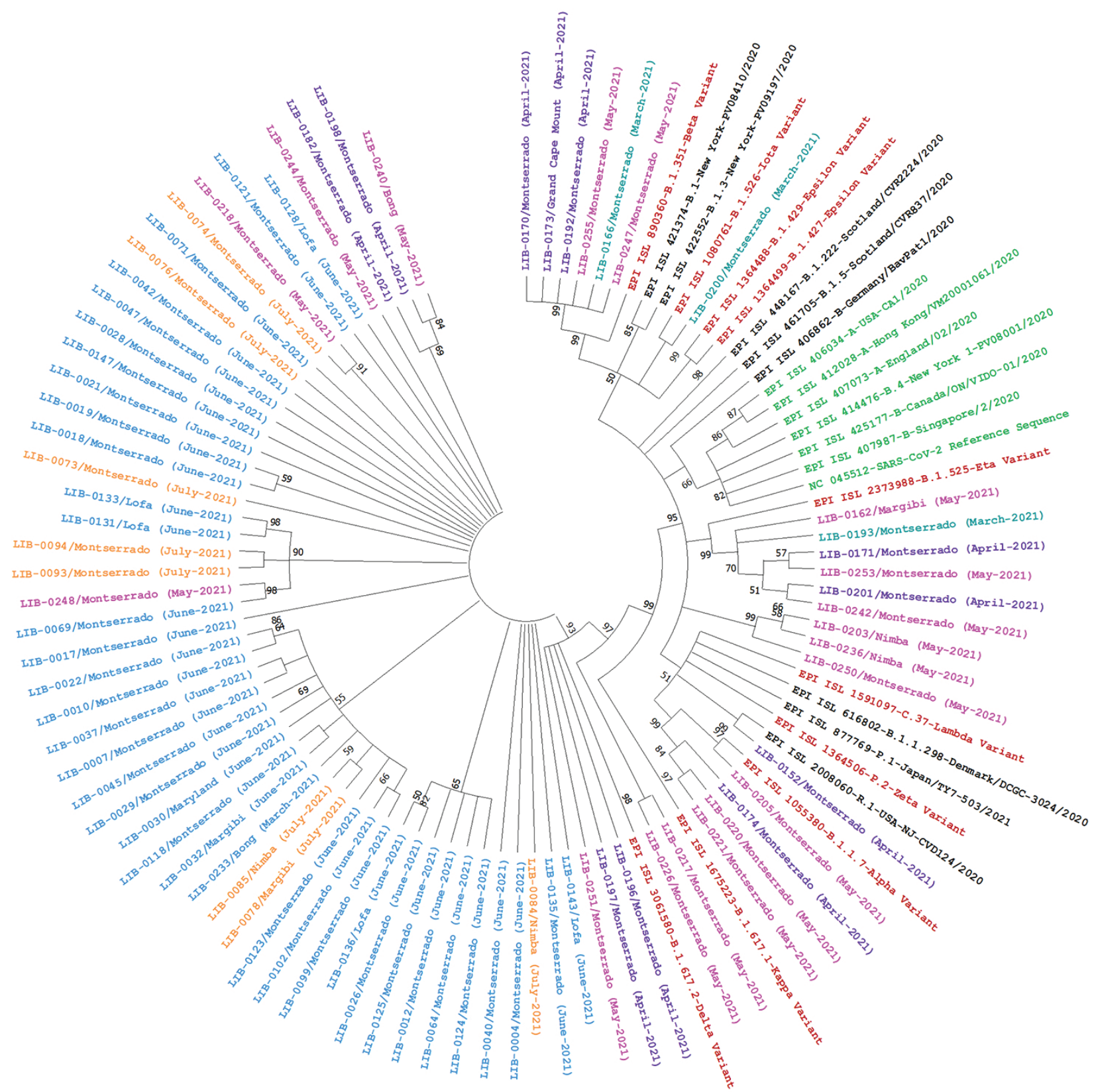

Figure. Phylogenetic analysis of 77 nasopharyngeal swab samples collected during coronavirus disease case surge, Liberia, MarchJuly 2021, and reference sequences. We created a maximum-likelihood nucleotide phylogenetic tree of the complete polyprotein coding region by using MEGA X (https://www.megasoftware.net), with a bootstrap value of 100 and and used Tamura-Nei 93 (TN93) as a substitution model with a discrete gamma distribution $(+G)$ for evolutionary rate; the rate variation model allowed some sites to be evolutionarily invariable $(+\mathrm{I})$. Numbers along the branches are bootstrap values of 100 bootstrap resamplings. Teal indicates samples collected in March 2021; purple indicates samples collected in April 2021; pink indicates samples collected in May 2021; blue indicates samples collected in June 2021; orange indicates samples collected in July 2021; brown indicates variants of concern or variants of interest; black indicates other circulating variants; green indicates severe acute respiratory syndrome coronavirus 2 reference sequence and other early parental sequences from 2020. 


\section{References:}

1. Worldometer. COVID-19 coronavirs disease data, Liberia [cited 2021 Sep 26]. https:/ / www.worldometers.info/ coronavirus/country/liberia

2. US Food and Drug Administration. Accelerated emergency use authorization (EUA) summary the Triplex CII-SARSCoV-2 rRT-PCR test updated 9/14/2020 [cited 2021 Sep 15]. https:/ / www.fda.gov/media/137983/download

3. Mishra N, Ng TFF, Marine RL, Jain K, Ng J, Thakkar R, et al. Antibodies to enteroviruses in cerebrospinal fluid of patients with acute flaccid myelitis. MBio. 2019;10:e01903-19. https://doi.org/10.1128/mBio.01903-19

4. GISAID. Tracking of variants [cited 2021 Sep 15]. https://www.gisaid.org/hcov19-variants

5. Next-Strain. Real-time tracking of pathogen evolution [cited 2021 Sep 15]. https:/ / nextstrain.org

6. Kumar S, Stecher G, Li M, Knyaz C, Tamura K. MEGA X: Molecular Evolutionary Genetics Analysis across computing platforms. Mol Biol Evol. 2018;35:1547-9. https:/ / doi.org/ 10.1093/molbev/msy096

7. Lopez Bernal J, Andrews N, Gower C, Gallagher E, Simmons R, Thelwall S, et al. Effectiveness of Covid-19 vaccines against the B.1.617.2 (Delta) variant. N Engl J Med. 2021;385:585-94. https://doi.org/10.1056/NEJMoa2108891

8. Alizon S, Haim-Boukobza S, Foulongne V, Verdurme L, Trombert-Paolantoni S, Lecorche E, et al. Rapid spread of the SARS-CoV-2 Delta variant in some French regions, June 2021. Euro Surveill. 2021;26.2100573. https:// doi.org/ 10.2807/1560-7917.ES.2021.26.28.2100573

9. Creech CB, Walker SC, Samuels RJ. SARS-CoV-2 vaccines. JAMA. 2021;325:1318-20. https://doi.org/10.1001/ jama.2021.3199

Address for correspondence: Nischay Mishra, Center for Infection and Immunity, Columbia University, 722 W 168th St, New York, NY 10032, USA; email: nm2641@cumc.columbia.edu

\section{Real-Time Projections of SARS-CoV-2 B.1.1.7 Variant in a University Setting, Texas, USA}

\author{
Kaitlyn E. Johnson, ${ }^{1}$ Spencer Woody, ${ }^{1}$ Michael \\ Lachmann, Spencer J. Fox, Jessica Klima, Terrance \\ S. Hines, Lauren Ancel Meyers
}

Author affiliations: University of Texas at Austin Department of Integrative Biology, Austin, Texas, USA (K.E. Johnson, S. Woody, S.J. Fox, L. Ancel Meyers); Santa Fe Institute, Santa Fe, New Mexico, USA (M. Lachmann); University of Texas at Austin Office of the Vice President for Research, Austin (J. Klima); University of Texas at Austin Department of Population Health, Austin (T.S. Hines)

DOI: https://doi.org/10.3201/eid2712.210652

We used the incidence of spike gene target failures identified during PCR testing to provide an early projection of the prevalence of severe acute respiratory syndrome coronavirus 2 variant B.1.1.7 in a university setting in Texas, USA, before sequencing results were available. Findings from a more recent evaluation validated those early projections.

Tdentification of the highly transmissible novel seIvere acute respiratory syndrome coronavirus 2 (SARS-CoV-2) variant B.1.1.7 (Alpha variant) in the United Kingdom raised concerns for renewed pandemic surges worldwide (1,2). B.1.1.7 likely arrived in the United States by October 2020 (1); it was first detected in December 2020 and declared the dominant strain in April 2021, as projected in January 2021 (3). However, the regional prevalence of B.1.1.7 was largely unknown in early 2021 because of limited molecular surveillance for SARS-CoV-2 (4). To provide local situational awareness at that pivotal moment in the coronavirus disease (COVID-19) pandemic, we estimated the prevalence of B.1.1.7 on the basis of 17,003 student SARS-CoV-2 PCR test results reported through the Proactive Community Testing Program at the University of Texas (UT; Austin, Texas, USA), a large public university located in a metropolitan area with a population $>2$ million, during January 16February 12, 2021 (K.E. Johnson et al., unpub. data, https://doi.org/10.1101/2021.03.05.21252541. Those early estimates were subsequently validated by using PCR data through April 9, 2021.

Mutations in the B.1.1.7 spike protein result in a failure to detect the spike gene probe in standard SARS-CoV-2 quantitative reverse transcription PCR (qRT-PCR). In estimating the prevalence of B.1.1.7 from local quantitative PCR data, we initially assumed US estimates for the proportion of spike gene target failures (SGTF) attributable to B.1.1.7 (4) and, in our retrospective analysis, update that proportion on the basis of local sequencing data. We used a Bayesian model to estimate the local growth rate of B.1.1.7 among all SARS-CoV-2 infections and applied a compartmental susceptible-exposed-infected-recovered model of SARS-CoV-2 transmission to project the effect of B.1.1.7 on future COVID-19 prevalence.

${ }^{1}$ These authors contributed equally to this article. 